was found in 12/401 (3\%) children and 58/401 (14.5\%) suffered from constipation. Lastly, a significant percentage of children presented innocent heart murmur (86/401 - 21.4\%). Conclusions Our experience demonstrate that there are many comorbidities that influence the prognosis and the response to the therapy in these children.

\section{P563 PROFILE OF PAEDIATRIC PATIENTS WITH URINARY TRACT INFECTION}

Otilia Frasinariu*, Violeta Streanga, Aniela Rugina, Nicolai Nistor. University of Medicine and Pharmacy Grigore T. Popa, lasi, Romania

10.1136/archdischild-2019-epa.897

Background and aims Urinary tract infection (UTI) is one of the most common pediatric infections. The aim of the study was to evaluate the clinical-epidemiological profile of children with UTI.

Methods The study group consists in 87 children, 55 girls and 32 boys, aged 3 months - 13 years, hospitalised in a Paediatric Clinic during 16 months, diagnosed with urinary tract infections. Diagnostic criteria were the presence of pyuria and/ or bacteriuria on urinalysis and the presence of more than 100.000 colony-forming units (CFU) per $\mathrm{mL}$ of a uropathogen.

Results The distribution of patients by age groups showed a higher prevalence in the age-groups 1-12 months, 24,14\%, and 7-14 years, 29,89, than in children 1-3 years age old, $19,84 \%$ or $3-7$ years age old group, $18,09 \%$, without statistical significance. Depending on the environment, $73,6 \%$ of patients were from urban areas and $22,4 \%$ from rural areas. The incidence of infections was higher in autumn-winter periods, 52\% of cases. Analyzing the way of admission, we found an increased number of the patients sent by the family doctor (63.22\%), followed by patents referred to the hospital in the emergency department, $27.59 \%$ of the patients and $5.75 \%$ of the patients were transferred from another hospital. The etiology of infections was Escherichia coli in $49.43 \%$ of cases, followed by Proteus mirabilis (4.60\%), Enterobacter aerogenes (3.45\%), Staphilococcus epidermidis (3.45\%), Enterococcus faecalis $(5.75 \%)$, Pseudomonas aeruginosa (1.15\%), Streptococcus faecalis $(4.60 \%)$, Candida albicans $(2.30 \%)$. The risk factors were present in $54 \%$ of our patients: $5,74 \%$ presented renal litiasis, 4,6\% duplicated renal collecting system, 10,3\% hidronefrosis, 9,2\% vesico-ureteral reflux, 2 patients neurogenic bladder, 3 patients chronic constipation, 1 patient coalescence of small labia.

Conclusions In urinary tract infection, E. coli is remaining the most prevalence etiological factor in our population: The prevalence is bigger in patients with a malformative risk factor.

\section{\begin{tabular}{|l|l}
\hline P564 A QUALITY IMPROVEMENT PROJECT ON ORAL FOOD \\
\hline
\end{tabular} CHALLENGE}

Amruta Fulmali*, Halima Sadiya Gumi. North Devon District Hospital, Barnstaple, Barnstaple, UK

10.1136/archdischild-2019-epa.898

Introduction This is a Quality Improvement project done in North Devon District Hospital, Barnstaple, UK (level 1 unit) to find out compliance with a Food challenge protocol and to improve our current practice.

We used a food challenge protocol from Royal Devon and Exeter Hospital as a standard protocol.

Methods Total number of food challenges audited were 25 .

All the notes were inspected against following standards: Age, Gender, Type of allergen Challenged, written consent, Time lag between Skin Prick test (SPT) and Food challenge, Risk assessment done or not, allergy focused documentation of clinical examination before and after challenge, Observations before and after challenge, outcome of challenge.

Results Main population was between age 1-5 years with Female to male ratio of 1.08 . We carried out a variety of challenges; majority being baked eggs and milk.

We found out quite a few gaps in our practice.

1. An informed written consent was not taken for any of the challenges.

2. None of the parents were given information leaflet about food challenge.

3. Last skin prick test result was documented in Only $3 / 25$ (12\%) food challenges.

4. Last antihistamine use was documented before 1/25 (4\%) food challenge.

5. None of the children with high risk challenges had IV line done beforehand.

6. $80 \%$ of the time we didn't examine the child after food challenge was complete hence outcome of the challenge was not documented clearly.

7. $44 \%$ of our cohort of population had to wait for $>2$ months after negative SPT disparate recommended waiting time of $2-4$ weeks.

Conclusions Currently we are not following recommended protocol for a food challenge. If a proper protocol is not followed during a food challenge then there are high chances of failed food challenges and anaphylaxis. This might lead to elevated parental anxiety and needless exclusion of food from diet which may lead to essential vitamin/mineral deficiency.

Actions Following this audit, we formed pro-forma which includes all the relevant information required before the food challenge and is easy to follow.

If we gather all the relevant information; then a Junior doctor and nurse will feel comfortable when carrying out a food challenge and outcome of challenge will be accurate and therefore less or no false positives.

Single page pro forma will save time required to clerk these patients on a busy day on the ward.

\section{P565 VALIDATION OF NOVEL GROWTH-PROMOTING AND GROWTH-SUPPRESSING GENES IN NEUROBLASTOMA CELLS}

Dalia Hammouche*, Andrew Stoker. ICH GOSH UCL, London, UK

10.1136/archdischild-2019-epa.899

Background Neuroblastoma is the most common extra-cranial embryonal tumour in children. Treatment outcomes and fiveyear survival rates are very poor, thus for improved results there is a need to understand its genetic and molecular drivers.

Aim This project focused on novel genes that can potentially be targeted to induce growth suppression or increase neural differentiation in Neuroblastoma cells. 